\title{
ITER ECRH Upper Launcher Torus Diamond Window - Prototyping, Testing and Qualification
}

\author{
Sabine Schreck ${ }^{\mathrm{a}}$, Gaetano Aiello ${ }^{\mathrm{a}}$, Andreas Meier ${ }^{\mathrm{a}}$, Dirk Strauss ${ }^{\mathrm{a}}$, Ryosuke Ikeda ${ }^{\mathrm{b}}$, \\ Yasuhisa Oda $^{\mathrm{b}}$, Keishi Sakamoto ${ }^{\mathrm{b}}$, Koji Takahashi ${ }^{\mathrm{b}}$ and Theo Scherer ${ }^{\mathrm{a}}$ \\ ${ }^{a}$ Karlsruhe Institute of Technology, Institute for Applied Materials, Association KIT-EURATOM, \\ P.O. Box 3640, D-76021 Karlsruhe, Germany \\ ${ }^{b}$ Japan Atomic Energy Agency (JAEA), Plasma Heating Technology Group, \\ 801-1 Mukoyama, Naka, Ibaraki 311-0193 Japan
}

The diamond window assembly is part of the ITER primary vacuum boundary and acts as the first tritium barrier and therefore it is classified as Safety/Protection Important Component (SIC/PIC). It consists of an ultra-low loss CVD diamond disk mounted in a system of metallic parts (copper/steel) and has to fulfil adequate transmission capability for high power mm-waves.

High power RF experiments with a $1^{\text {st }}$ window prototype had shown parasitic heating due to small gaps in the housing. After a design optimization directed to the mm-wave properties, the parasitic excitations of oscillations have been avoided in a $2^{\text {nd }}$ prototype. This one is equipped with inserted waveguide structures, which cover gaps in the metallic structure of the window housing. From high power RF-measurements with a $0.86 \mathrm{MW} / 100 \mathrm{~s}$ pulse a loss tangent of $7.1 \times 10^{-6}$ could be estimated, corresponding to an increase of temperature of only $120 \mathrm{mK}$ between inlet and outlet of the cooling system.

The diamond window assemblies cannot be entirely covered by codes and standards. To comply with the French safety regulations, instead an ad-hoc qualification programme is required, being developed in the framework of a contract between KIT and F4E. A new prototype $\left(3^{\text {rd }}\right)$ will be built, which is designed to fit to the single HELICOFLEX sealed waveguide structures of the ex-vessel mm-system of the EC Upper Launcher (UL). The testing program ranges from mechanical to vacuum tests up to dielectric loss measurements at low and high power. A clear definition of the testing requirements and of the acceptance criteria is necessary as well as a complete documentation of the process.

Keywords: ITER, Upper, Launcher, Diamond, Window

\section{Introduction}

The ITER ECRH system consists of EC launchers at the equatorial and upper level. The four upper launchers (UL), which have the main purpose to drive local current with the aim to control the plasma instabilities (mitigate NTMs), are connected via transmission lines to the ITER gyrotrons providing up to $20 \mathrm{MW} \mathrm{mm}$-wave power at $170 \mathrm{GHz}[1,2]$. The system requires the use of ultra low loss CVD (chemical vapour deposition) diamond windows, designed to be compatible with the transmission of mm-wave power of up to $1.5 \mathrm{MW}$ into the launcher and therefore into the vacuum vessel and to the plasma. The torus window assembly and the isolation valve form the barriers in the first confinement system for tritium and are part of the primary vacuum boundary. The diamond window assembly consists of a synthetic polycrystalline diamond disk produced by 'Microwave Plasma Assisted (MPA)' chemical vapour deposition with a low loss tangent of $\tan \delta<2 \times 10^{-5}$ mounted in a system of metallic parts (copper/steel).

The level of dielectric loss and its lateral homogeneity over a diamond disk depend critically on growth conditions. At KIT Karlsruhe, a dedicated measurement facility (FABRY-PEROT-resonator) is available. The distribution of the dielectric loss of the bare disk is parameterized with a hemi-spherical measurement set-up (a spherical mirror and a plane mirror) and the loss tangent at the center of the disk is evaluated in a symmetric resonator with two spherical mirrors. With the latter device also complete window assemblies can be measured.

Different prototypes of the window assembly have already been manufactured to verify the capability of the windows considering mm-wave transmission and the cooling efficiency of the indirect cooling concept.

High power RF (radio frequency) experiments of the prototypes have been carried out at the JAEA gyrotron test facility. In a first campaign the applied maximum power was $520 \mathrm{~kW}$ at $170 \mathrm{GHz}$. IR thermography and thermocouples were used to investigate the temperature distribution over the diamond disk and in the housing.

Experiments of the first prototype with a real nonGaussian input beam profile (ca. 90\% Gaussian content) show the influence of high frequency higher order modes (parasitic modes) introduced by excitation of oscillations in construction-conditioned small cavities in the window assembly and therefore an additional heating of the window housing $[3,4]$. An advanced design (prototype II) with inserted waveguides, covering the small gaps between copper cuff and waveguides of the former prototype, succeeded in avoiding the parasitic excitations. A comparable design with inserted corrugated waveguides has been developed by JAEA, with the difference of the use of a direct cooling concept [5].

Within an ongoing contract with F4E another new prototype is being built. The complete qualification and testing program for the window is under development and tests will be performed with this prototype III, which 
is expected to represent the final design of the ITER ECRH UL torus window assembly.

\section{Design development and experimental investigations of window prototypes}

\subsection{Prototype I}

In Fig. 1 the CAD design of the window prototype I is shown. The diamond disk is brazed to a copper cuff and integrated into a metallic housing. The cooling circuit is formed by two symmetric cooling chambers which are separated by a vacuum segment at the edge of the diamond disk, which means that the coolant is not in direct contact with the diamond disk (indirect cooling).

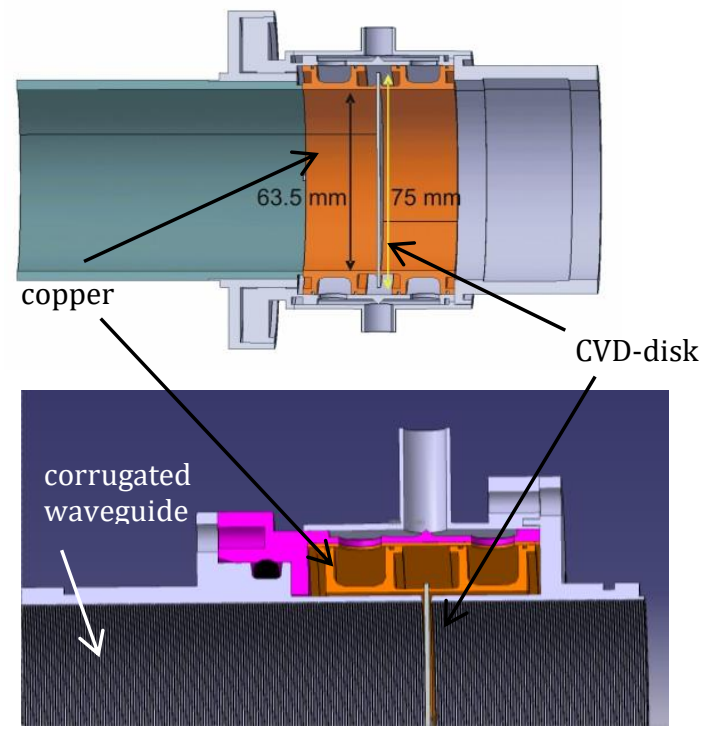

Fig. 1. a) Prototype I CAD design, b) Prototype II CAD design with inserted corrugated waveguides.

The bare disk of prototype I had shown a low effective loss at the centre of $\tan \delta=0.9 \times 10^{-5}$ at $170 \mathrm{GHz}$. After brazing to the copper cuffs a loss tangent of $\tan \delta=3.4 \times 10^{-5}$ had been observed.

High power RF experiments (JAEA) have been performed with mm-wave power up to $520 \mathrm{~kW}$. One side of the window unit was evacuated and mounted to the supply waveguide from the gyrotron source while the opposite side was in air, which allowed IR thermography. After free space propagation the RF power was dissipated in a CCR dummy load.

In Fig. 2 the time-temperature dependence (a) and the temperature distribution (b) during a long RF pulse at $520 \mathrm{~kW} / 30 \mathrm{~s}$ are shown. The temperature distribution at the window surface was not a centred peaked distribution and therefore it was assumed that the RF wave propagated with some percentage of higher order modes. Additional heating of the housing (point $\mathrm{C}$ and thermocouple measurement) was observed and is a sign of microwave absorption. The gap between copper and steel was about $100 \mu \mathrm{m}$. In the given case of a mode mixing in the microwave beam additional parasitic cavity heating can be expected and is an obvious source for the additional heating of the housing. a)

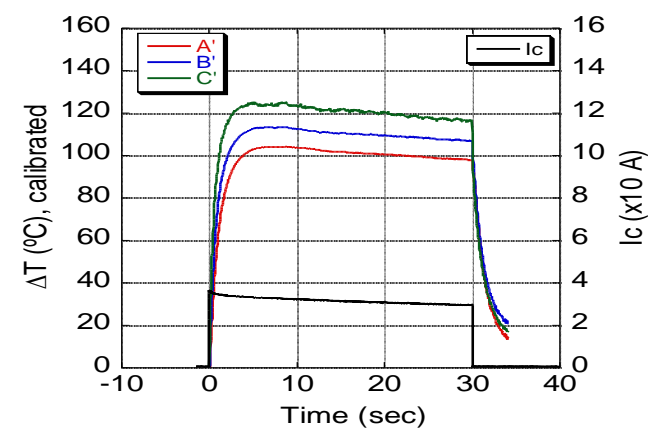

b)

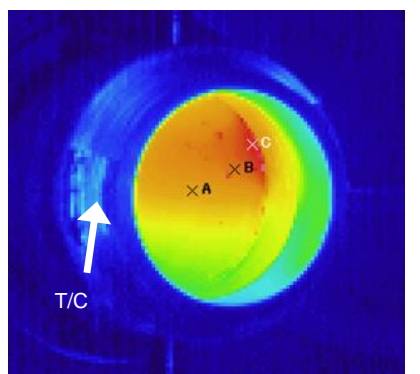

Fig. 2. IR measurements for prototype I during pulse operation $(520 \mathrm{~kW} / 30 \mathrm{~s})$ : a) Temperature/time dependence and b) IR image (at the end of the pulse; AVIO TVS-8500 IR camera)

\subsection{Prototype II}

The second prototype of the ITER torus CVD diamond window is equipped with a HELICOFLEX sealed vacuum flange and inserted corrugated waveguides which cover small gaps of $50-100 \mu \mathrm{m}$ between the copper cuffs and the SS waveguide (Fig. 1b).

Using the symmetric resonator $(170 \mathrm{GHz})$ a very low effective loss of $\tan \delta=0.4 \times 10^{-5}$ was determined in the centre of the bare disk and after the brazing the value was increased only slightly to $\tan \delta=0.55 \times 10^{-5}$.

The objective of the RF experiments at JAEA/Naka Japan was the demonstration of high power transmission capability of prototype II (improved mm-wave design) and the investigation of any abnormal heating in the window housing. As before visual monitoring of the window by video camera and temperature measurement by an infrared camera was carried out during pulse operation. Accordingly, the operation was carried out with one side of the window being in air and not in vacuum, which is why the power was limited to about $500 \mathrm{~kW}$ due to the risk of arcing. A cooling system was attached to the window (water flow 5.5 1/min @ 10 ${ }^{\circ} \mathrm{C}$ ). Two thermo-couples allowed the measurement of the temperature difference between the inlet and the outlet of the cooling water. Experiments with different pulse durations were performed from several ms up to $20 \mathrm{~s}$. No significant temperature increase on the diamond window unit was measured, neither for the IR measurement nor for the thermocouples. In Fig. 3 an IR image during a $500 \mathrm{~kW}$ pulse is shown. As usual, visible "star lights" were observed at the disk surface, which are well known, and caused by the typical hydrogen surface bonds. Due to the free air operation a brick shielding was applied (pink bottom picture part) to absorb the reflection power from the RF load. The results indicate that the bricks are heated up by the reflection power, but all temperatures in the window are low enough to operate at $\mathrm{CW}$ and are reduced compared to those measured with prototype I. 


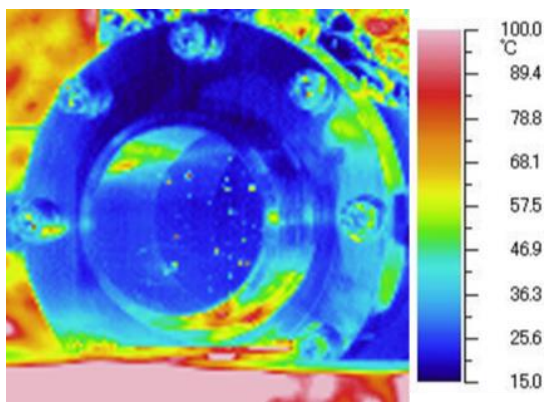

Fig. 3. IR image of prototype II during pulse operation $\sim 500 \mathrm{~kW} /$ up to $20 \mathrm{~s}$

The deposited power into the window could be coarsely estimated from the $\Delta \mathrm{T}$ difference between inlet and outlet of the cooling water to only $55 \mathrm{~kW}$. With the transmission power of $498 \mathrm{~kW}$ the loss tangent was estimated to be $\tan \delta=8.4 \times 10^{-6}[6]$.

It can be concluded that the sensitivity to parasitic higher order modes, which was observed for prototype I, is minimized for prototype II. Therefore the "modeproblem" was considered as solved. As a consequence the temperature in prototype II is much lower compared to prototype I.

Nevertheless a second campaign for high power RF measurements of prototype II took place in Jan./Feb. 2014 with the aim to reach operation with higher power and longer pulses. During these experiments the window assembly was installed in the transmission line system evacuated from both sides. Table 1 summarizes microwave power levels and pulse lengths used. By using the phase retrieve analysis method [7], the $\mathrm{HE}_{11}$ mode content of the RF beam was estimated to $91.2 \%$.

Table 1. High power experiments for prototype II at JAEA (averaged gyrotron power and pulse length)

\begin{tabular}{|l|c|c|}
\hline No. & Power [kW] & Pulse length [s] \\
\hline 1 & 490 & 200 \\
\hline 2 & 600 & 150 \\
\hline 3 & 620 & 300 \\
\hline 4 & 720 & 200 \\
\hline 5 & 800 & 224 \\
\hline 6 & 820 & 100 \\
\hline
\end{tabular}

Fig. 4 shows typical recorder traces of the experimental parameters for a $720 \mathrm{~kW} / 200 \mathrm{~s}$ pulse. The power at the CCR dummy load, the RF signal and the temperature rise of the window cooling water are monitored.

From the measurement and calibration procedure in JAEA the input mm-wave power at the position of the diamond disk can be evaluated. Corresponding to that, a correlation of the temperature increase of the cooling water during pulse operation and power can be obtained. In Fig. 5 the increase of temperature ( $\Delta \mathrm{T}$ between inlet and outlet with $10 \mathrm{l} / \mathrm{min}$ water flow) is plotted for four power values. The increase of temperature over power shows a linear behaviour. For $1 \mathrm{MW} / 170 \mathrm{GHz}$ pulse operation the increase is expected to be below $150 \mathrm{mK}$, which indicates the extreme low loss tangent of the diamond disk. Observing the IR pictures of the window/waveguide system, the window housing, which is connected to the cooling circuit has a temperature below $25^{\circ} \mathrm{C}$ and is therefore not critical.

From these high power RF experiments the loss tangent can be evaluated to $\tan \delta=7.1 \times 10^{-6}$. This value is in very good agreement with the measured loss tangent at the disk centre in the low power measurement in the FABRY-PEROT - resonator.

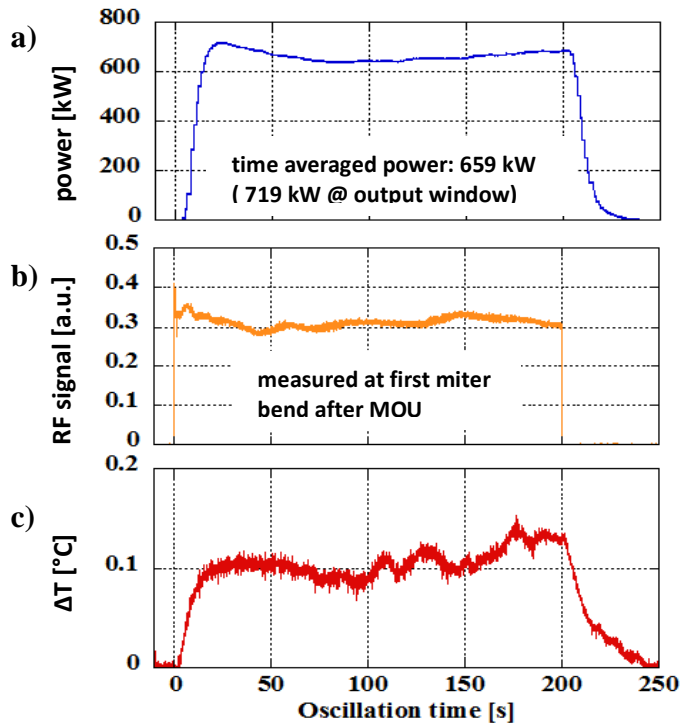

d) IR picture at end of pulse:

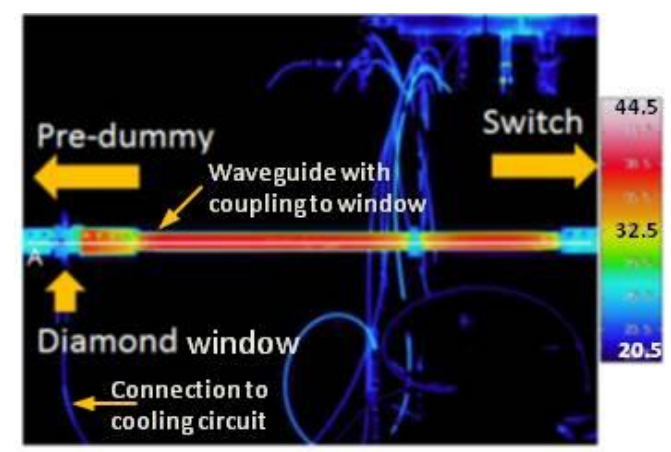

Fig. 4. RF pulse $720 \mathrm{~kW} / 200 \mathrm{~s}$ : (a) Power at CCR dummy load, b) RF signal, c) Temperature rise $\Delta \mathrm{T}$ of window coolant water and d) IR image of the window/waveguide system.

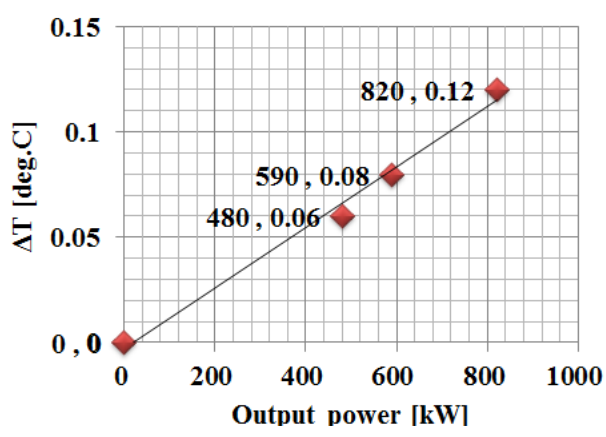

Fig. 5. Linear temperature increase $\Delta \mathrm{T}$ versus microwave power at the window unit.

\section{3 (Future) Prototype III}

The new prototype III is expected to have the final design for the ITER ECRH UL torus window assembly. It consists of a $1.111 \mathrm{~mm}$ thick diamond disk brazed to two oxygen-free high conductivity (OFHC) copper cuffs with embedded cooling channels allowing the indirect cooling of the disk. Two nickel rings, named spacer rings, connect the cuffs to corrugated stainless steel waveguides which are inserted into the cuffs leaving a $100 \mu \mathrm{m}$ gap with the diamond disk. This waveguide 
system allows suppressing parasitic oscillations in the small cavities of the unit. The design of the new prototype was optimized by FEM analyses for the required load conditions and combinations. In general, the design strategy was to have a very rigid outer frame which withstands the external loads acting on the unit (e.g. baking, seismic, plasma disruption events) while the thin copper cuffs brazed to the diamond allow the indirect cooling of the disk. Prototype III will be equipped additionally with four diagnostic steel pipes which connect the unit to different diagnostic systems, for instance to detect any potential tritium leakage.

One main scope of the prototype work is to verify the implementation of the different manufacturing steps. The main challenging part during assembly is the brazing process which is carried out at about $800-900^{\circ} \mathrm{C}$ whereupon the temperature of the brazed parts (disk and cuffs) is decreased down to room temperature.

The new prototype will be qualified via a sophisticated testing program.

\section{Testing and Qualification program}

The window unit is part of the first ITER confinement system and thus it has the most stringent requirements in the ITER safety (SIC1 / PIC1), quality, vacuum, seismic and tritium classifications. As the design, the manufacturing and the qualification of the diamond window cannot be completely covered by standard codes, a specific qualification program is to be developed. Within this program acceptance criteria for clearly defined tests have to be determined, which guarantee the overall functionality of the component, especially the safety function.

The testing program for the window assembly can be split in different parts. Selected tests are executed by the manufacturer(s) of the window. Other tests have to be performed by qualified labs, e.g. dielectric properties measurements and seismic tests. Particular tests are required for the bare disk, for the disk already brazed to the copper cuffs and for the complete assembly.

The tests range from standard tests for geometry control, qualification of brazing by computer tomography, vacuum and leakage tests to mm-wavetransmission tests. All testing conditions have to be compliant with the event driven loads expected during the lifetime of an ITER window. Tests are required to demonstrate the safety function and the high performance of the window. All tests have to be supplemented by an extensive documentation, including description of test procedure, data acquisition plan, calibration of the measurement setups, certification of used materials, error and uncertainty analysis etc.. An appropriate documentation is necessary for the manufacturing process of the window (equipment description, process parameters, environmental conditions, material certificates, etc.).

The overall testing and qualification plan needs to respect and fulfil the requirements of nuclear safety, as postulated in the ITER Preliminary Safety Report. The approach required for testing and qualification of components with no existing codes and standards is mainly characterized by establishing of damage limits for the expected loads/load combinations and by comparing the test results against these limits. The complete procedure needs to be accompanied by supporting analysis.

\section{Conclusions and Outlook}

The tests performed with the prototype II proved the mm-wave design of the window. It features a very good transmission capability, a very low loss and the heating of the window can be rated as not critical. The combination of the high quality of the disk and of the window design has been shown to be very robust also in case of relatively low quality of the EC beam (ca. $90 \%$ Gaussian content).

Further work will be directed to the proof of the mechanical integrity of the window under the specific ITER load conditions and its compliance with safety requirements. Therefore various tests need to be performed with the new prototype III and as a result also the qualification program for the serial window production will be established.

\section{Acknowledgments}

This work is/was supported by Fusion for Energy (F4E) (contracts No. OPE-140 and OPE-467) and by the German Ministry of Research and Education (BMBF, 03FUS0010).

The views and opinions expressed herein reflect only the author's views. F4E/BMBF are not liable for any use that may be made of the information contained therein.

\section{References}

[1] D. Strauss et al., Progress of the ECRH Upper Launcher design for ITER // Fusion Engineering and Design 89 (2014) 1669-1673.

[2] T. Omori et al., Overview of the ITER EC H\&CD system and its capabilities // Fusion Engineering and Design 86 (2011) 951-9541.

[3] T. A. Scherer et al., Experimental and theoretical thermal analysis of CVD diamond windows for the ITER Upper launcher // Infrared, Millimeter and Terahertz Waves (IRMMW-THz), 2008.

[4] T. A. Scherer et al., Recent upgrades of the ITER ECRH CVD torus diamond window design and investigations of dielectric diamond properties // Electron Cyclotron Emission and Electron Cyclotron Resonance Heating (EC-16), 2011: pp. 396-400.

[5] K. Takahashi et al., High power millimeter wave experiment of torus diamond window prototype for ITER EC H\&CD system // Fusion Engineering and Design 88 (2013) 85-93.

[6] T. A. Scherer et al., Experimental analysis of the inserted waveguide CVD diamond window Infrared // Millimeter and Terahertz Waves (IRMMW-THz), 2011.

[7] O. Yasuhisa et al., Measurement of RF Transmission Mode in ITER Relevant EC H\&CD Transmission Line // J. Infrared Milli. Terahz. Waves (2010) 3: 949-957. 